\title{
Sticklet: An End-User Client-Side Augmentation-Based Mashup Tool
}

\author{
Oscar Díaz and Cristóbal Arellano \\ ONEKIN Research Group, University of the Basque Country (UPV/EHU), \\ San Sebastián, Spain \\ \{oscar.diaz, cristobal.arellano\}@ehu.es \\ http://www.onekin.org/
}

\begin{abstract}
A critical aspect of mashup tools for end users is to come up with an intuitive metaphor. Sticklet is an augmentation-based mashup tool that conceives websites as walls where you can fix $H T M L$ fragments (sticky notes) from other websites. Notes are contextualized to the hosting website, i.e. location, parameter passing and layout should be harmonized to those of the website. A set of declarative constructs are available to declaratively specify complex sticky notes. Sticklet is realized as an internal DSL in JavaScript that capitalizes on browser weavers (e.g. Greasemonkey $(G M))$. Being full-fledged GM scripts, Sticklet benefits from the sharing repositories (e.g. www.userscripts.org) or management utilities (e.g. activation, installation, edition) available for $G M$.
\end{abstract}

\section{Motivation}

We address a special kind of mashuping known as Web augmentation [2]. Web Augmentation is to the Web what Augmented Reality is to the physical world: layering relevant content/layout/navigation over the existing Web to customize the user experience. For instance, when rendering a book at Amazon, it could be useful to know the prices/comments for this book at other online bookshops, or to directly check if this book is available at your library, all without leaving Amazon. Traditionally, this is achieved through JavaScript (JS) using browser weavers (e.g. Greasemonkey). To date, over 43 million of downloads of Greasemonkey scripts ground the vitality of this movement. However, these efforts are hindered by being programming intensive and prone to malware. We strive to open Web Augmentation to users other than $J S$ programmers. To this end, we developed Sticklet, an internal DSL in JavaScript which targets $J S$-ignorant users. Users are expected to be computer-literate (e.g. able to write an Excel formula).

\section{Sticklet}

The lifecycle of a Sticklet expression (hereafter referred to as a "sticklet") includes three main stages: definition, deployment and enactment.

Definition. Sticklet conceives the Web as a wall to be decorated with stickers (i.e. HTML fragments dynamically obtained from other websites). The pair (wall, 


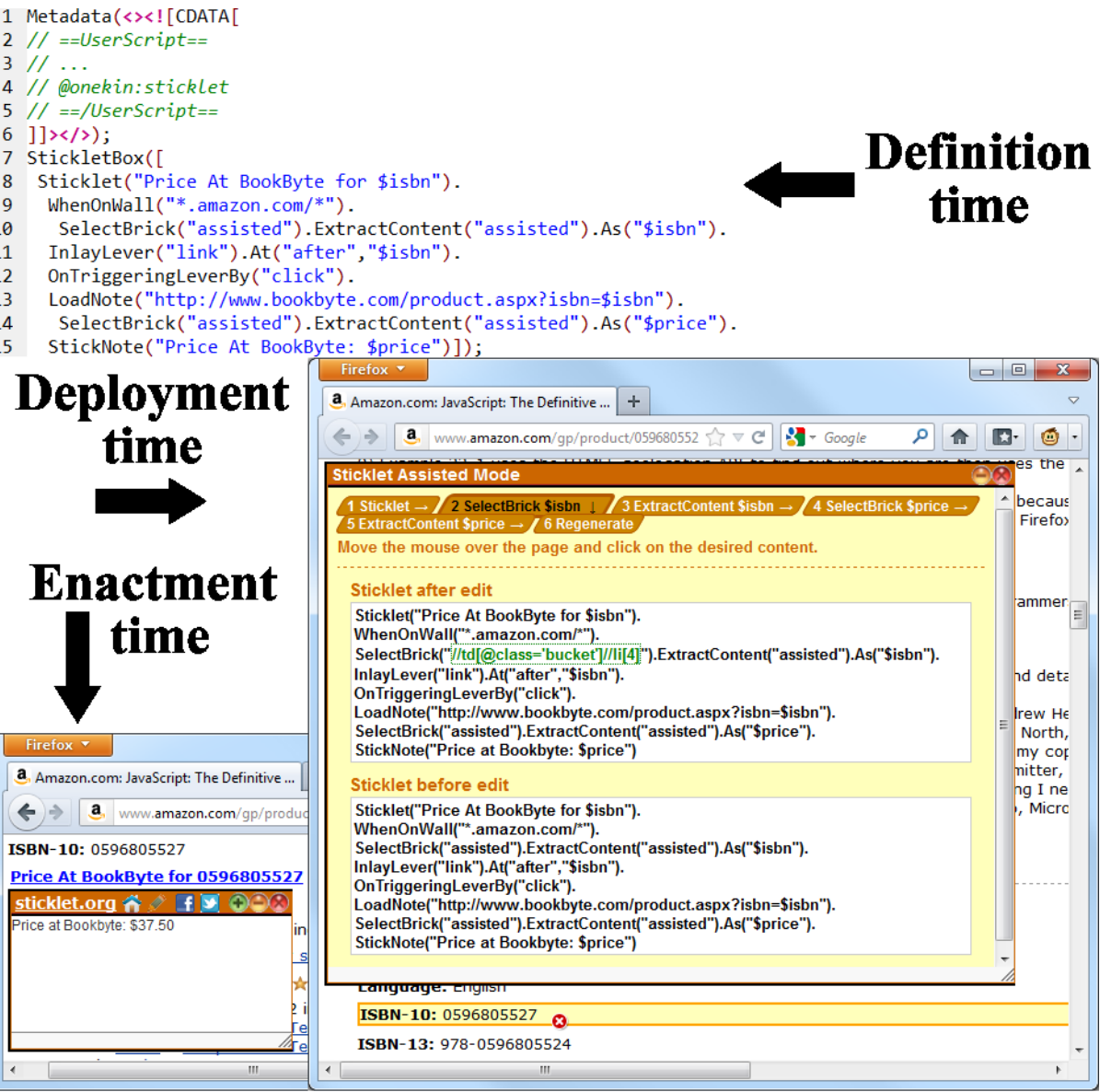

Fig. 1. Sticklet lifecycle: definition, deployment and enactment

sticker) conforms an augmentation unit, i.e. a sticklet. Consider fixing stickers in Amazon with the prices of the current book at other online bookshops. This is a popular JS script known as BookBurro which accounts for over 900 lines of $J S$ cod 1 . The sticklet counterpart is shown in Figure 1. The constructs of the DSL include: walls, bricks, notes and levers. Walls (line 9) can be regarded as "views" upon existing websites. A wall comprises those websites whose URLs match a given regular expression (WhenOn Wall clause). The scope of the sticklet is defined by its wall as well as the existence of some bricks. For our sample problem, the wall expands along those Amazon pages that hold an ISBN brick. Bricks (lines 10 \& 14). They are named nodes upon HTML documents which are worth singularizing for either data extraction, scoping or layering purposes. A brick holds (1) an XPath to pinpoint the node (SelectBrick clause), (2) a

${ }^{1}$ BookBurro is available at http://userscripts.org/scripts/source/1859.user.js 
regular expression to extract the node's content (ExtractContent clause), and (3), the brick's name (As clause). Notes (line 15) are expressions that combine text and bricks (StickNote clause). Bricks can be obtained from the wall as well as from URL-addressable services (LoadNote clause, line 13). For the sample problem, a request is made to BookByte where URL parameters are obtained from previously extracted bricks (e.g. \$isbn). The outcome is used to pinpoint a new brick: \$price. Finally, bricks from different sources are used to conform the note (StickNote clause). Notes might be readily stuck as soon as the user enters the wall or displayed on demand by acting on a lever. Levers (line 11) permit to obtain notes on demand. Levers are named after the sticklet (e.g. "Price At BookByte for \$isbn") where the brick content is resolved at runtime. Levers are positioned according to bricks. For our sample problem, a lever (realized as a link) is inlayed after the brick \$isbn. On acting upon the lever (in this example through a "click"), a URL-addressable service is enacted. For our sample case, on clicking, the BookByte request is conducted; next, the book price is obtained and finally, the note is rendered.

Deployment. Being Sticklet an internal DSL, sticklets do behave as traditional Greasemonkey $(G M)$ scripts, e.g. the file extension is .user.js. Hence, sticklets' deployment is achieved in the very same way as $G M$ scripts: drag\&drop on Firefox. However, unlike GM scripts, sticklets postpone the definition of some of its clauses till deployment time. Specifically, "assisted-valued" clauses are resolved at deployment time through the help of the Sticklet engine. Specifically, $X P a t h$ and regular expressions are heuristically obtained by interacting with the user. Sticklet intersperses a grid-like structure on top of the current DOM tree. As the user moves the cursor around the screen, the DOM node under the current cursor location is highlighted. By clicking, the user feeds the XPath inferring algorithm with the selected node as an example, while Sticklet highlights all the nodes that fulfilled the extraction pattern generated so far. This process is iterated till the desired nodes are selected. The process ends by re-generating the sticklet but now fully resolved and ready for enactment.

Enactment. Sticklets are automatically executed by GM when on the wall. Pages are augmented with the sticklet supplement as soon as they are loaded and lever triggered. Besides $G M$ itself, this requires the previous installation of the Sticklet engine 2 . Over 20 sticklets are available at http://userscripts.org/users/Sticklet.

\section{Related Work}

Platypus is a Visual Programming Tool [4. It obtains full-fledged JavaScript code for Greasemonkey using a graphical toolbar. Users directly act upon the current page through the Platypus toolbar to obtain the GM script. Platypus is a neat tool for its purpose: changing a web page based on the page itself. On the downside, visual tools might restrict the expressiveness to facilitate

\footnotetext{
2 https://addons.mozilla.org/addon/Sticklet/
} 
code generation (e.g. in Platypus, no page other than the current page can be accessed).

Chickenfoot illustrates the API approach [1]. An API introduces some abstractions that shelters users from how these abstractions are implemented but without leaving the hosting language. Chickenfoot is more expressive than Sticklet. Sticklet just focuses on a special kind of customization: augmentation. But this limited focus permits to come up with a self-contained, domain-oriented vocabulary, and to stick to this vocabulary. Unlike APIs, DSLs have to do without resorting to the underlying language. Sticklets can only contain Sticklet terms. No general JS sentences are permitted. Although this limits expressivity, it brings understandability and trustworthiness ("malware free by construction").

MashMaker illustrates a hybrid approach [3. A distinctive aspect is that programmers and end-user asynchronously collaborate to come up with the augmentation. A MashMaker project encompasses three artefacts: the data extractor (graphically defined), the augmentation widget (which is separately coded in JavaScript), and the so-called "mashup" (graphically defined). The "mashup" links the two previous artefacts so that the widget is fed from the extractor. A library of widgets is made available by programmers to end users. This introduces two actors during augmentation: widget programmers and enduser "linkers". By contrast, Sticklet relies on a single user.

\section{Conclusions}

We introduce Sticklet, a textual DSL for Web Augmentation. Sticklet is based on $J S$ but limits $J S$ generality for the sake of learnability and understandability. First evidences indicate that users with no $J S$ background can easily build their own sticklets, and, not less important, understand someone else's sticklets, hence promoting sharing and collaboration.

\section{References}

1. Bolin, M., Webber, M., Rha, P., Wilson, T., Miller, R.C.: Automation and Customization of Rendered Web Pages. In: Proceedings of the 18th Annual ACM Symposium on User Interface Software and Technology, Seattle, USA, pp. 163-172 (October 2005)

2. Bouvin, N.O.: Unifying Strategies for Web augmentation. In: Proceedings of the 10th ACM Conference on Hypertext and Hypermedia, Darmstadt, Germany, pp. 91-100 (February 1999)

3. Ennals, R., Brewer, E.A., Garofalakis, M.N., Shadle, M., Gandhi, P.: Intel Mash Maker: Join the Web. SIGMOD Record 36, 27-33 (2007)

4. Turner, S.R.: Platypus (2005), http://platypus.mozdev.org 